\title{
My personal mutanome: a computational genomic medicine platform for searching network perturbing alleles linking genotype to phenotype
}

Yadi Zhou ${ }^{1 \dagger}$, Junfei Zhao ${ }^{2,3+}$, Jiansong Fang ${ }^{1+}$, William Martin ${ }^{1}$, Lang Li', Ruth Nussinov ${ }^{5,6}$, Timothy A. Chan ${ }^{1,7,8,9,10}$, Charis Eng ${ }^{1,8,9,10,11}$ and Feixiong Cheng ${ }^{1,8,9^{*}}$ (i)

\author{
* Correspondence: chengf@ccf.org \\ ${ }^{\dagger}$ Yadi Zhou, Junfei Zhao and \\ Jiansong Fang contributed equally \\ to this work. \\ 'Genomic Medicine Institute, Lerner \\ Research Institute, Cleveland Clinic, \\ Cleveland, OH 44195, USA \\ ${ }^{8}$ Department of Molecular Medicine, \\ Cleveland Clinic Lerner College of \\ Medicine, Case Western Reserve \\ University, Cleveland, OH 44195, \\ USA \\ Full list of author information is \\ available at the end of the article
}

\begin{abstract}
Massive genome sequencing data have inspired new challenges in personalized treatments and facilitated oncological drug discovery. We present a comprehensive database, My Personal Mutanome (MPM), for accelerating the development of precision cancer medicine protocols. MPM contains 490,245 mutations from over 10, 800 tumor exomes across 33 cancer types in The Cancer Genome Atlas mapped to 94,563 structure-resolved/predicted protein-protein interaction interfaces ("edgetic") and 311,022 functional sites ("nodetic"), including ligand-protein binding sites and 8 types of protein posttranslational modifications. In total, 8884 survival results and 1, 271,132 drug responses are obtained for these mapped interactions. MPM is available at https://mutanome.lerner.ccf.org.
\end{abstract}

Keywords: Mutanome, Protein-protein interaction, Edgetic, Nodetic, Somatic mutations, Precision cancer medicine

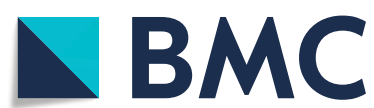

(C) The Author(s). 2021 Open Access This article is licensed under a Creative Commons Attribution 4.0 International License which permits use, sharing, adaptation, distribution and reproduction in any medium or format, as long as you give appropriate credit to the original author(s) and the source, provide a link to the Creative Commons licence, and indicate if changes were made. The images or other third party material in this article are included in the article's Creative Commons licence, unless indicated otherwise in a credit line to the material. If material is not included in the article's Creative Commons licence and your intended use is not permitted by statutory regulation or exceeds the permitted use, you will need to obtain permission directly from the copyright holder. To view a copy of this licence, visit http://creativecommons.org/licenses/by/4.0/. The Creative Commons Public Domain Dedication waiver (http://creativecommons.org/publicdomain/zero/1.0/) applies to the data made available in this article, unless otherwise stated in a credit line to the data. 
computational approaches can only identify a small portion of the pathogenic variants with the high confidence that is required for clinical decision making. Identification and prioritization of causal and clinically actionable mutations is essential to understand their roles in tumorigenesis and disease progression, discover new biomarkers, and offer biologically relevant drug targets [7].

Genes and proteins do not function independently, but in complex, interconnected networks and pathways [8-10]. The human interactome is a network of proteins (nodes) connected by their physical interactions (edges) (Fig. 1 and Additional file 1: Fig. S1). Mutations perturb the network either by directly altering the normal functions of the proteins ("nodetic" effect), such as via posttranslational modification and ligand-binding, or by altering the protein-protein interactions (PPIs) ("edgetic" effect). Theoretically, in the human interactome, nodetic effect refers to the effect that a mutation directly knockout or knockdown a gene/ protein function and consequently removing the protein and all its edges [8,9]; alternatively, mutation effects can also be PPI specific, causing removal or gain of specific PPIs, known as edgetic effect [8-10]. Nodetic and edgetic network perturbations by mutations have been shown to promote tumorigenesis and disease progression [11] and result in altered patient survival and drug responses. Our previous studies have shown that in cancer, somatic missense mutations tend to be enriched at protein functional sites such as protein-ligand binding sites [12], protein allosteric sites [13], and phosphorylation sites [14]. Investigation of the

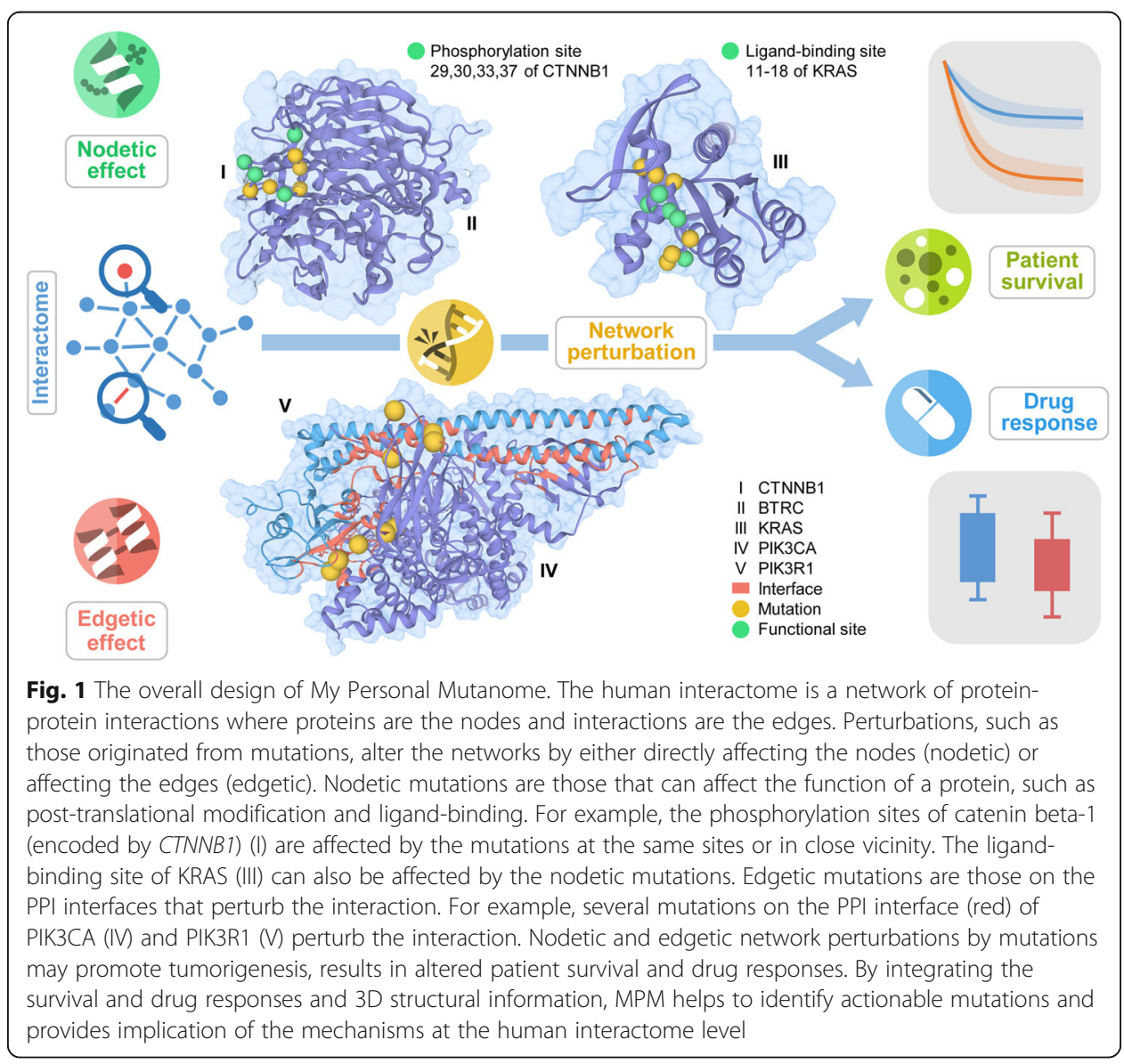


nodetic effects of mutations could help uncover likely driver mutations with mechanistic implications and offer personalized drug treatments [6]. Studies have shown that disease-related mutations tend to localize in PPI interfaces and perturb the interactions of the same mutated protein with multiple partners [10, 15]. Recent ongoing community efforts have completed the mapping of the human interactome and provided the increasing availability of structural genomic information on PPIs from diverse sources including the PDB [16], Interactome INSIDER [17], and Interactome3D [18]. These protein structural genomic resources offer unexpected opportunities for accelerating interpretation of biological and functional consequences of cancer mutations for precision cancer medicine from systems biology perspectives $[6,19]$. In our recent study, we found that somatic missense mutations were highly enriched in PPI interfaces compared to non-interfaces via analysis of over 10,000 whole exomes across 33 cancer types [20]. We further showed that PPI interface mutation analysis provided likely causal relationships in tumorigenesis and experimentally validated functional effects of PPI interface mutation using a systematic binary interaction assay [8-10] and cell line-based functional assays [20]. In summary, all previous observations from our groups and other studies provide functional proof-of-concept of both nodetic and edgetic effects of somatic mutations in human cancer. These results motivate us to develop a systems biology tool for querying such nodetic and edgetic mutations in the human interactome, which will be valuable for identifying novel functional mutations/ genes, drug targets, and pharmacogenomics biomarkers for precision cancer medicine.

We therefore developed My Personal Mutanome (MPM), a comprehensive database of nodetic and edgetic effects of somatic mutations across 33 cancer types/subtypes. Figure 2 summarizes the main data entities and their relationships, as well as eight main questions addressed by the data and tools provided by MPM. We integrated 490,245 somatic mutations, 121,172 physical PPIs, and 535,182 functional sites composed of 8 varieties: acetylation (43,764), malonylation (4476), methylation (14,649), O-linked glycosylation (4228), phosphorylation $(276,738)$, succinylation (1665), ubiquitination $(100,246)$, and ligand binding (89, 416). We systematically mapped all the mutations to 94,563 PPIs and 311,022 functional sites. For the human interactome, we combined data from three sources to build a comprehensive PPI interface database. Using statistical methods, we systematically identified putative SMEs (Significantly Mutated Edges, also termed oncoPPIs) which harbor a statistically significant excess number of somatic missense mutations at PPI interfaces (see Methods). We then performed survival and drug response analysis for these mappings. MPM offers three interactive visualization tools that provide $3 \mathrm{D}$ views of somatic mutations in the context of the human interactome network (nodetic and edgetic) with their clinical (survival) and drug responses. MPM is expected to facilitate the identification of actionable mutations for tumorigenesis and personalized treatments at the human interactome level. Collectively, it offers network-based diagnosis and pharmacogenomics approaches to understand complex genotype-phenotype relationships and therapeutic responses in the clinical settings. MPM is available at https:// mutanome.lerner.ccf.org. 


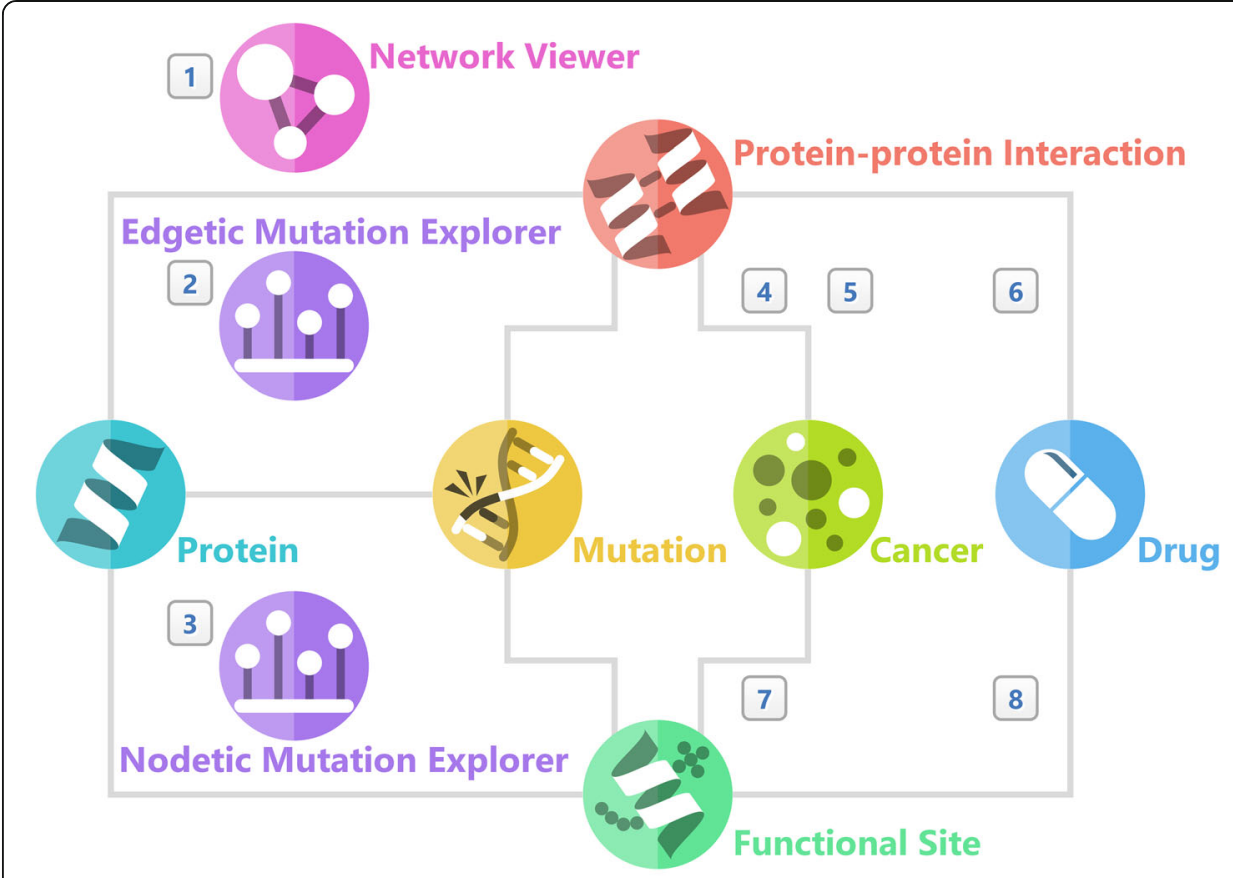

\begin{tabular}{|c|c|}
\hline \multicolumn{2}{|r|}{ Questions Addressed by My Personal Mutanome } \\
\hline \multirow[t]{2}{*}{1} & What are the SMEs for a certain protein? \\
\hline & How many interface mutations do they have? \\
\hline \multirow[t]{2}{*}{2} & What are the interface mutations for a PPI? \\
\hline & How many cases do they have in different cancer types? \\
\hline \multirow[t]{3}{*}{3} & What are the functional sites of a protein? \\
\hline & What mutations can potentially affect them? \\
\hline & Are they significantly mutated in certain cancer types? \\
\hline 4 & Is the PPI significantly interface mutated? \\
\hline 5 & Is the interface mutated PPI associated with patient survival? \\
\hline 6 & Does the interface mutated PPI cell line react differently to drugs? \\
\hline 7 & Is the mutated functional site associated with patient survival? \\
\hline 8 & Does the cell line with the mutated functional site react differently to drugs? \\
\hline
\end{tabular}

Fig. 2 Information architecture and questions addressed by My Personal Mutanome. This figure illustrates the several main entities (such as protein and drug) and their relationships (indicated by edges) in My Personal Mutanome. Users can browse through all the information by following the architecture shown here. Three visualization tools were developed: "Network Viewer," which shows the subnetwork of the significantly mutated edges of a certain protein; "Nodetic Mutation Explorer," which bridges the mutations to the functional sites; and "Edgetic Mutation Explorer," which displays the mutations on the protein-protein interaction interfaces. Both "Nodetic" and "Edgetic" come with a built-in protein 3D structure viewer. Drug responses and cancer patient survival were computed for the nodetic and edgetic mutations. Numbers indicate the questions addressed by the information and visualization provided by My Personal Mutanome

\section{Construction and content}

\section{Data collection}

\section{Genes and proteins}

Gene information was retrieved from HGNC (https://www.genenames.org/) [21]. Protein information was downloaded from UniProt (https://www.uniprot.org/uniprot/) [22]. Gene and protein mapping were downloaded using the "Retrieve/ID mapping" tool from 
UniProt. A total of 21,759 proteins with somatic mutations from TCGA database were mapped to 19,149 protein coding genes from HGNC.

\section{Human protein-protein interactome}

We used the human protein-protein interactome from our previous studies [20, 23]. Briefly, high-quality PPIs were assembled from 15 commonly used databases that include five types of evidence: yeast-two-hybrid system, protein 3D structures, literaturederived kinase-substrate interactions, literature-derived signaling networks, and affinitypurification mass spectrometry.

\section{PPI interfaces}

PPI interfaces were combined using three sources: PDB (http://www.rcsb.org/) [16], ECLAIR (http://interactomeinsider.yulab.org/) [17], and Interactome3D (https:// interactome3d.irbbarcelona.org/) [18]. PDB provides many resolved 3D structures that contains both interacting proteins in some PPIs. ECLAIR and Interactome3D utilizes machine learning-based approaches and homology modeling to predict PPI.

\section{Post-translational modification sites}

Seven types of post-translational modifications sites (acetylation, malonylation, methylation, O-linked glycosylation, phosphorylation, succinylation, ubiquitination) were downloaded from four databases: dbPTM (http://dbptm.mbc.nctu.edu.tw/) [24], PhosphoSitePlus (https://www.phosphosite.org/homeAction.action) [25], Phospho.ELM (http://phospho.elm.eu.org/) [26], and PTMD (http://ptmd.biocuckoo.org/) [27].

\section{Ligand-binding site}

We downloaded the ligand-binding site data from BioLiP (https://zhanglab.ccmb.med. umich.edu/BioLiP/) [28], which offers high-quality manually curated ligand-protein binding information.

\section{Somatic mutations and cancer patient information}

We downloaded 10,861 human exomes (the tumor-normal pairwise somatic mutation data) across 33 cancer subtypes/types and their survival information from TCGA GDC Data Portal (https://portal.gdc.cancer.gov/). We integrated the results of four scoring methods for the evaluation of pathogenic impacts of the mutations. The sorting intolerant from tolerant (SIFT) and polymorphism phenotyping v2 (PolyPhen-2) were computed using ANNOVAR [29]. Combined Annotation Dependent Depletion (CADD) scores were downloaded from https://cadd.gs.washington.edu/ [30]. FoldX scores (change in structure stability between mutated and reference structure, ddG) were downloaded from http://www.mutfunc.com/ [31].

\section{Drug responses}

A total of 251 drugs tested in 1074 cancer cell lines with half maximal inhibitory concentration $\left(\mathrm{IC}_{50}\right)$ data points were downloaded from GDSC (http://www. cancerrxgene.org/) [32]. For each drug, we constructed a drug-response vector consisting of $n \mathrm{IC}_{50}$ values from treatment of $n$ cell lines. Then, drug-response vector was 
modeled as a linear combination of the tissue of origin of the cell lines, screening medium, growth properties, and the status of a genomic feature. A genomic featuredrug pair was tested only if the final drug-response vector contained at least three positive cell lines and at least three negative cell lines. Effect size was quantified through the Cohen's $d$ using the difference between two means divided by a pooled standard deviation for the data. The resulting $p$ values were corrected by Benjamini-Hochberg method.

\section{Nodetic and edgetic effects evaluation}

We first mapped all somatic mutations to the PPI interfaces and protein functional sites. Using the interface information, all mutations on a certain PPI were classified as either interface mutations or non-interface mutations. For functional sites, a 15- or 7amino acid window was applied for each site (from position -7 to +7 centered at the post-translational modification sites, and -3 to +3 at the ligand-binding sites) to screen for mutations as described in previous studies $[12,14]$.

For each type of functional site (e.g., phosphorylation site), we tested whether the mutations of gene $g_{i}$ in a certain cancer type are significantly enriched near the functional site. We computed the $p$ value using binomial distribution:

$$
P(X \geq k)=1-P(X<k)=1-\sum_{x=0}^{k-1}\left(\frac{T}{x}\right) p_{g_{i}}^{x}\left(1-p_{g_{i}}\right)^{T-x}
$$

where $T$ is the total number of mutations observed in the protein product of gene $g_{i}$, and $p_{g_{i}}$ is the estimated mutation rate for the window flanking this functional site under the null hypothesis. Using W to represent the window size and $L_{g_{i}}$ the length of protein product of gene $g_{i}, p_{g_{i}}$ was calculated as

$$
p_{g_{i}}=\frac{W}{L_{g_{i}}}
$$

Next, we computationally identified putative SMEs harboring a statistically significant excess number of missense mutations at PPI interfaces in pan-cancer analysis and individual cancer analysis under the null hypothesis that the mutations were randomly distributed on the sequences of two proteins of gene $g_{1}$ and $g_{2}$ in each PPI. Similarly, using $L_{g_{i}}$ to represent the length of protein product of gene $g_{i}$, we calculated the mutation rate in the interface of $g_{i}$ as

$$
p_{g_{i}}=\frac{L_{g_{i} *}}{L_{g_{i}}}
$$

where $L_{g_{i} *}$ is the interface length. After using binominal test to assess the significance of enrichment of mutations in the interfaces of $g_{1}$ and $g_{2}$, we used the product of two $p$ values $P_{1}$ and $P_{2}$ to represent the significance of mutation enrichment in this PPI interface. 


\section{Survival analysis}

Kaplan-Meier survival analysis (adjusting age, tumor stages, and other confounding factors) was performed with the patient survival data from TCGA using the R (3.6.3) (https://www.r-project.org/).

\section{Website implementation}

MPM was implemented with Python (3.7.2) (https://www.python.org/) framework Django (2.2.2) (https://www.djangoproject.com/) on the server backend. Django adopts a Model-Template-View pattern that decouples the database, content, and website logic, which allows rapid implementation of website features and provides high reusability of each component. SQLite (https://www.sqlite.org/) was used for the relational database. We decided to implement the views such that they respond to user requests with pure JSON format data. This architecture enables users to access all our data through user programs so that MPM can be integrated in their pipelines. HTML, CSS, and JavaScript were used for the frontend. The frontend was heavily programmed in JavaScript to offer the user a smooth experience with highly interactive visualization tools. AJAX was used to asynchronously retrieve data in JSON format and populates the web page on user requests. Network visualization was implemented using Cytoscape.js [33]. The PDB viewer was implemented based on PV [34]. Nodetic and edgetic mutation explorers were implemented with HTML canvas and JavaScript. MPM is hosted by the Cleveland Clinic Lerner Research Institute Computing Services.

\section{Utility and discussion}

\section{Database overview}

We have assembled and processed all the data, including 21,759 proteins, 490,245 somatic mutations, and 544,692 mutation cases (count excludes those neither mapped to PPIs nor functional sites), 121,172 PPIs, drug responses of 251 drugs tested in 1074 cancer cell lines, 41,843 PDBs, and 535,182 protein functional sites for protein-ligand binding and across 7 types of protein post-translational modifications (PTMs): acetylation, malonylation, methylation, o-linked glycosylation, phosphorylation, succinylation, ubiquitination (Table 1).

We mapped all the mutations to the PPIs and protein functional sites. We found that somatic missense mutations are significantly enriched in ligandbinding sites (Additional file 1: Fig. S2) and phosphorylation sites (Additional file 1: Fig. S3) compared to non-ligand-binding sites and non-phosphorylation sites, respectively, across all 33 cancer types/subtypes, which are consistent with our previous findings [12-14]. Survival analyses were performed by dividing patients into a wild-type group (not interface mutated for edgetic, or not mutated on the functional sites for the nodetic) and a mutant group. Drug response comparisons were conducted in the same manner (see Methods). For nodetic effects, we calculated the impact of mutations at the functional site type level to provide the user an overview of which type of functional site is significantly affected by somatic missense mutations in a specific cancer type. For edgetic effects, we performed oncoPPI test for all the PPIs in pan-cancer and 33 individual cancer 
Table 1 My Personal Mutanome database statistics

\begin{tabular}{|c|c|c|}
\hline \multicolumn{3}{|l|}{ Entities } \\
\hline Protein & 21,759 & \\
\hline \multirow[t]{3}{*}{ Mutation } & 490,245 & \\
\hline & Interface & 72,953 \\
\hline & Non-interface & 417,292 \\
\hline \multirow[t]{3}{*}{ Mutation case } & 544,692 & \\
\hline & Interface & 85,484 \\
\hline & Non-interface & 459,208 \\
\hline \multirow[t]{4}{*}{ PPI } & 121,172 from 3 sources: & \\
\hline & PDB & 4112 \\
\hline & Interactome3D & 2891 \\
\hline & ECLAIR & 114,169 \\
\hline \multirow[t]{9}{*}{ Functional site } & 535,182 in 8 types: & \\
\hline & Acetylation & 43,764 \\
\hline & Malonylation & 4476 \\
\hline & Methylation & 14,649 \\
\hline & O-linked glycosylation & 4228 \\
\hline & Phosphorylation & 276,738 \\
\hline & Succinylation & 1665 \\
\hline & Ubiquitination & 100,246 \\
\hline & Ligand binding & 89,416 \\
\hline TCGA & 11,315 cases in 33 cancer types & \\
\hline Drug & 251 & \\
\hline \multirow[t]{5}{*}{ PDB } & 41,843 & \\
\hline & 95,599 PDB chains for 6583 proteins & \\
\hline & 20,132 PDBs for 9589 PPI & \\
\hline & 5126 PDBs for 6482 PPI (hetero) & \\
\hline & 17,595 PDBs for 3107 PPI (homo) & \\
\hline \multicolumn{3}{|l|}{ Relations } \\
\hline & PPI (edgetic) & Protein functional site (nodetic) \\
\hline Mutation & 775,524 mapped interface mutations & 450,685 mapped mutations \\
\hline Cancer & $\begin{array}{l}7285 \text { survival analyses } \\
2,087,382 \text { oncoPPI tests }\end{array}$ & 1599 survival analyses \\
\hline Drug & 252,275 drug responses & $1,018,857$ drug responses \\
\hline
\end{tabular}

PPI protein-protein interaction, Hetero PPI PPI consisting of different proteins, Homo PPI PPI of the same protein

types/subtypes, which will enable the user to quickly search potentially mutationperturbing PPIs for a specific cancer type or pan-cancer from the human interactome.

PDBs were mapped to proteins at the residue level, enabling a simple and quick structural examination for each resolved residue. In addition, 5126 and 17,595 PDBs were mapped to 6482 heterodimers (hetero PPIs, two different proteins) and 3107 homodimers (homo PPIs, interaction of two identical proteins), respectively. When available, PDBs containing both proteins in a PPI are highlighted and prioritized for display, which helps to visualize the PPI interfaces to illustrate likely functional (hotspot or weak driver) mutations and their potential structural effects. 
All data and results have been integrated into a relational database. Our website utilizes the relations between several main entities (protein, mutation, PPI, functional site, and drug) to navigate the users to the information they are searching for, which is explained in the next section.

\section{Web interface}

The main web interface, Mutanome Explorer, is where users will perform data exploration and visualization in MPM. Mutanome Explorer is an all-in-one interface that utilizes the highly relational nature of the data. This permits smooth navigation of the
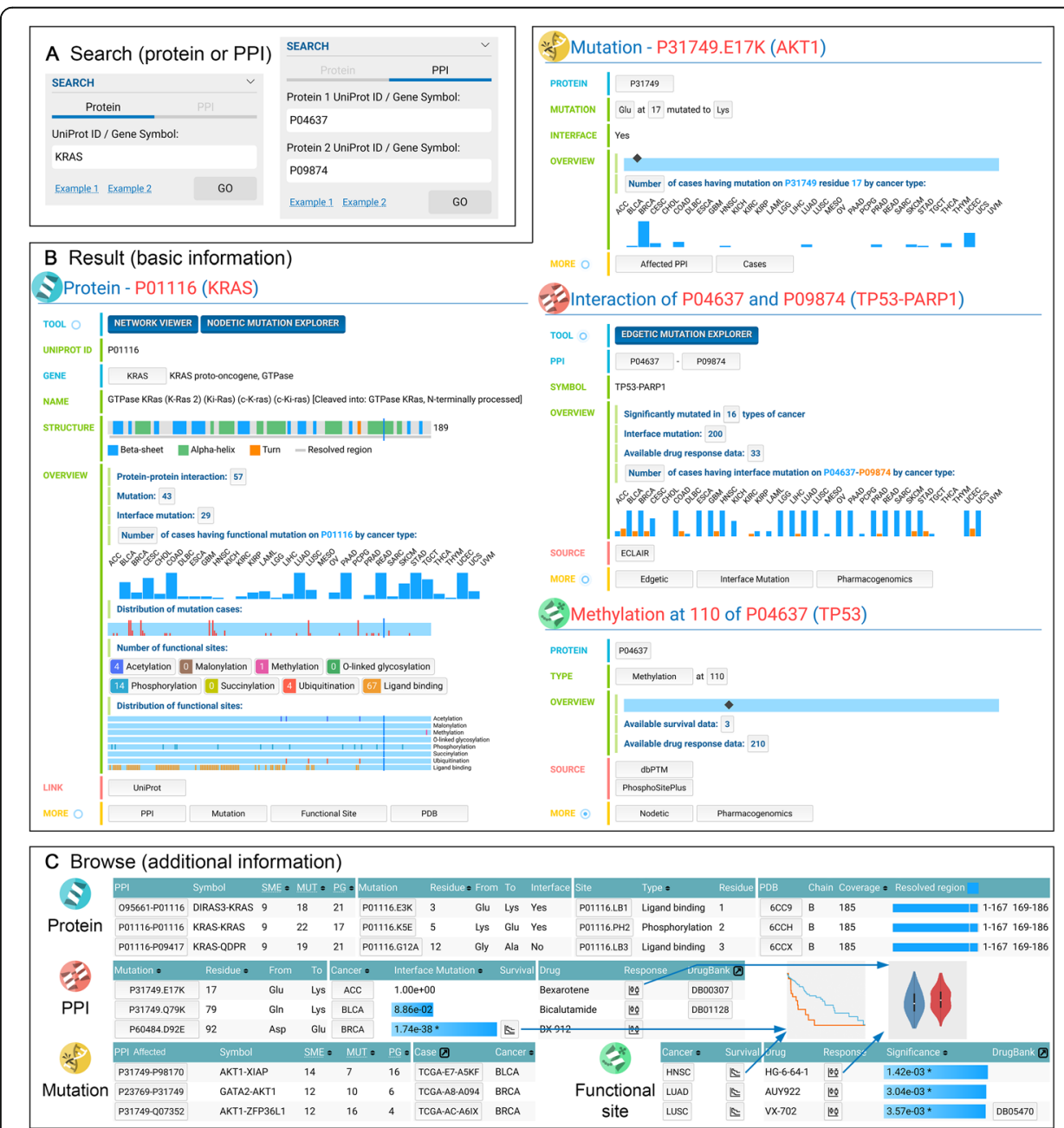

Fig. 3 Overview of the web interface. My Personal Mutanome has an all-in-one interface that allows users to search (a), view results (b, c), and visualize nodetic/edgetic mutations (Figs. 4 and 5) in the same web page, improving the smoothness in navigating through the database and removing the needs for switching between browser tabs. Users can use both UniProt ID and gene symbol to search for a protein or a specific PPI (a), which loads the basic information page (b). These pages provide an overview of the data that are available for the proteins, PPIs, mutations, and functional site in My Personal Mutanome. (c) Additional information such as all the mapped mutations for a specific PPI can be loaded on user request. Survival and drug responses are found by loading the "Edgetic" and the "Pharmacogenomics" information on the PPI and functional site pages. By clicking the buttons, users can browse through all the related information progressively 
data with minimal typing and searching. All data types are loaded onto the same web page, organized by tabs. Upon entering Mutanome Explorer, users will see an embedded help page with detailed instructions. The entry points to the database are protein and PPI searches (Fig. 3a), which accepts both UniProt ID and gene symbol. Then, a protein/PPI page is loaded. Protein, mutation, PPI, and functional site are the four major entities, with each having its own page (Fig. 3b). On each page type, several buttons at the bottom (in the "More" section) list the relevant related entities (Fig. 3c). For example, clicking "Mutation" on a protein page lists the mutations, and clicking a mutation in the list loads a new page for the mutation. Clicking the "Edgetic" and "Nodetic" shows the survival results for the PPI and functional site, respectively. The

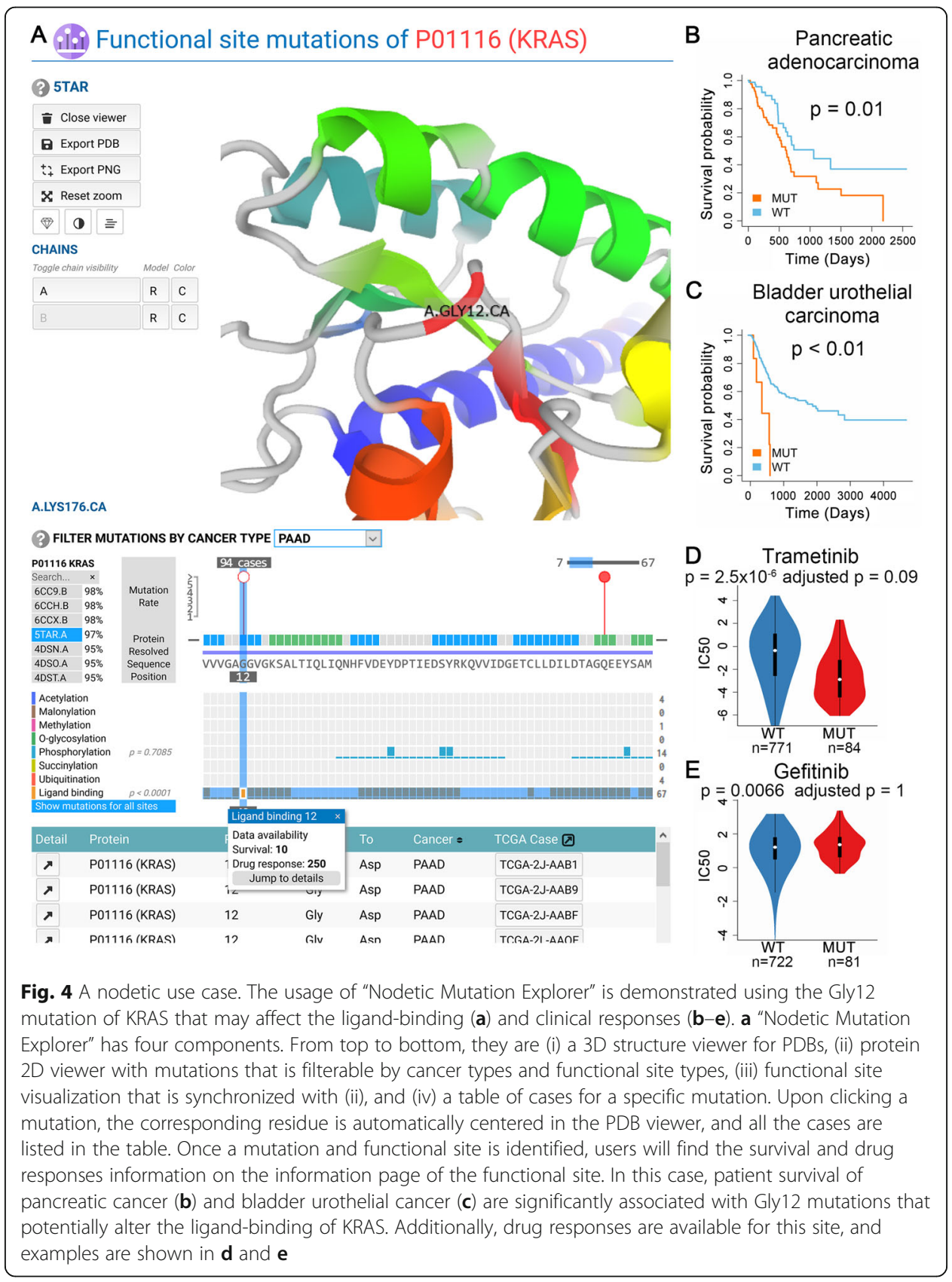


"Pharmacogenomics" button lists the drug response comparisons. Figure $3 \mathrm{c}$ shows an example for each type of list.

Several interactive visualization tools were implemented to facilitate the discovery of actionable mutations. "Network Viewer" helps to identify significantly mutated PPIs from the human interactome. "Nodetic Mutation Explorer" shows the mutations in a selected cancer type with 3D structure that potentially affects the functional sites (Fig. 4). "Edgetic Mutation Explorer" displays the interface mutations on a PPI with 3D structure and the mutation cases in various cancer types (Fig. 5). Usage of these tools is explained with two case studies next.

\section{Use case-nodetic example}

KRAS provides an example demonstrating how to use the "Nodetic Mutation Explorer" to identify clinically relevant and actionable functional site mutations. The KRAS gene

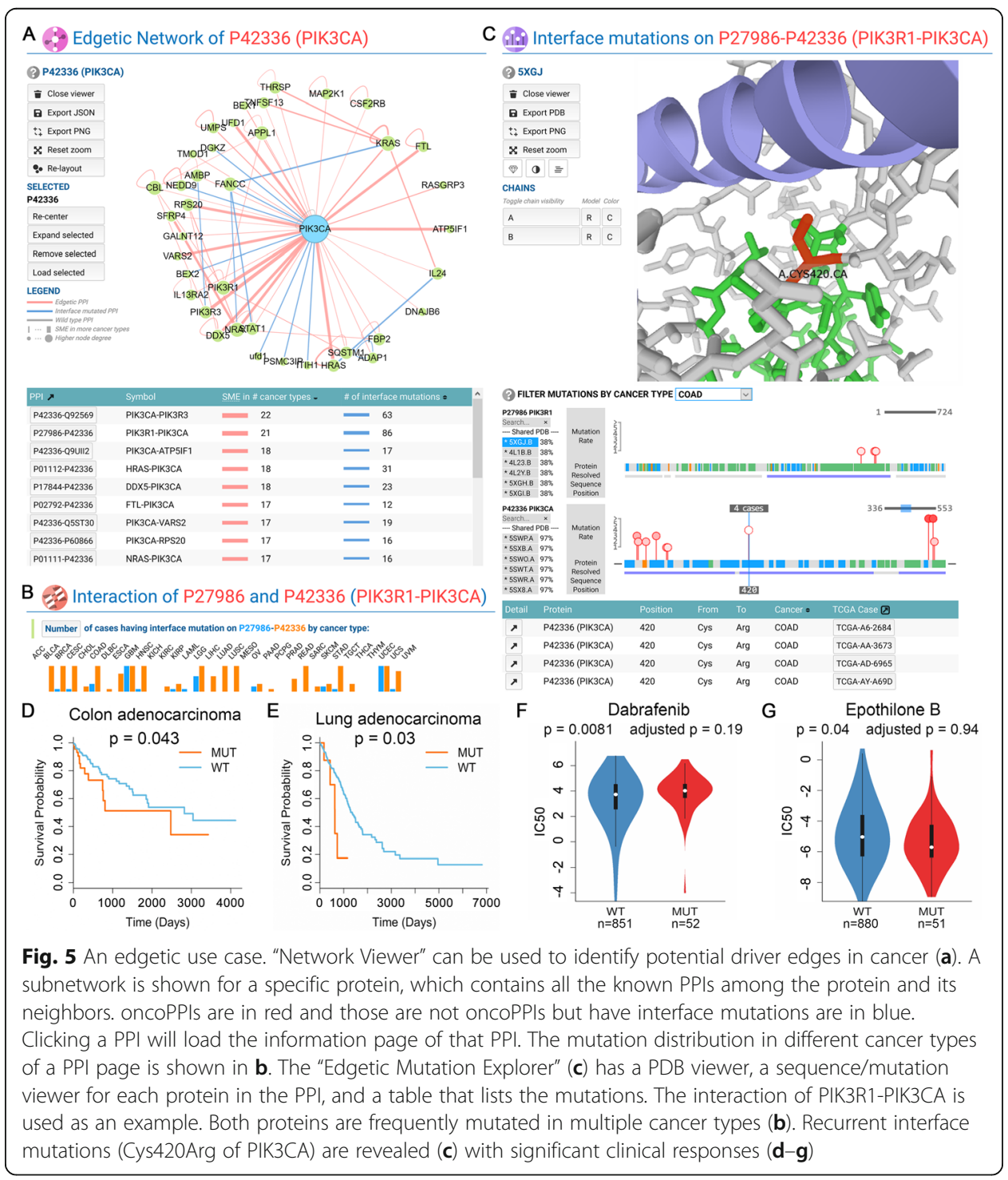


encodes a small ATPase that acts as a binary switch that controls signal transduction in cells [35]. It is one of the most mutated oncogenes in multiple cancer types [36-38].

We first searched for "KRAS" (or UniProt ID "P01116") in the protein search box to load the protein information page. In the "OVERVIEW" section, KRAS was most highly mutated in pancreatic adenocarcinoma (PAAD), colon adenocarcinoma (COAD), and rectum adenocarcinoma (READ). Out of the 185 PAAD TCGA cases, 101 cases (55\%) had mutations on KRAS that mapped to PPI interfaces or functional sites. For COAD and READ, the numbers are 163 out of 461 (35\%) and 49 out of 172 cases (28\%), respectively. Next, clicking on "NODETIC MUTATION EXPLORER," we entered the visualization tool. There are four sections in the tool (Fig. 4a). From top to bottom, they are (i) PDB viewer; (ii) protein sequence and mutation distribution viewer; (iii) functional site viewer organized by types, aligned with the protein sequence in (ii); and (iv) a table that shows the cases when a mutation is selected. The PDB file that has the highest coverage for KRAS was loaded automatically. By default, mutations in all cancer types are shown, but they can be filtered by a specific cancer type. We immediately noticed that Gly12 (439 cases), Gly13 (89 cases), Gln61 (50 cases), and Ala146 (29 cases) were the most mutated residues in pan-cancer. These mutations are established as oncogenic [39-41].

KRAS is strongly associated with pancreatic tumorigenesis [42, 43]. Having the highest mutation rate of KRAS compared to other cancer types, PAAD was selected to search the mutations. Ligand-binding sites were significantly affected by mutations (Fig. 4a, orange row, $p<0.0001$ ). Gly12 and Gln61 had 94 and 7 mutations cases, respectively, in PAAD. These mutation sites are also ligand-binding sites. Clicking the orange rectangle for ligand-binding site 12 opens the information page of this site. Examining the survival analysis results, the Gly12 mutations of ligand-binding site 12 is significantly associated with patient survival in PAAD (Fig. $4 \mathrm{~b}, p=0.01$ ). These mutations were also associated with bladder urothelial carcinoma (BLCA) patient survival (Fig. 4c, $p<0.01$ ). Next, by examining the drug response results, we found that mutations on ligand-binding site 12 altered several drugs' responses. Trametinib is a MEK inhibitor that has been approved by FDA for cancer treatment [44]. Trametinib had a lower $\mathrm{IC}_{50}$ in mutant cell lines (Fig. $4 \mathrm{~d}, p=2.5 \times 10^{-6}$ ), showing that Gly12 mutant cell lines were more sensitive to trametinib. This is consistent with the findings in a previous study [39]. In addition, there have been a number of clinical trials involving trametinib alone [45] or in combination with other drugs [46] for non-small cell lung cancer. Another drug, gefitinib, targets the epidermal growth factor receptor (EGFR) by inhibiting the tyrosine kinases associated with EGFR [47]. It has been approved for non-small cell lung cancer treatment. KRAS mutations are associated with a lack of sensitivity to gefitinib [48]. Gefitinib showed a higher $\mathrm{IC}_{50}$ in mutant cell lines (Fig. 4e, $p=0.0066$ ), confirming that KRAS mutants are more resistant to gefitinib.

\section{Use case-edgetic (oncoPPIs) example}

The interaction between the two phosphatidylinositol 3-kinase (PI3K) subunits provides an example of a mutation edgetic effect and the "Network Viewer" and "Edgetic Mutation Explorer." PI3K pathway, which regulates multiple cellular 
events such as cell growth, apoptosis, and survival [49], is frequently dysregulated in cancer. PIK3CA is also one of the most commonly mutated genes [50]. The PI3K $\alpha$ isoform is composed of two subunits: the catalytic subunit p110 $\alpha$ encoded by the PIK3CA gene and the regulatory subunit $\mathrm{p} 85$ encoded by the PIK3R1 gene.

In the PIK3CA page, PIK3CA is highly mutated (153 mutations and 1278 mutant cases), in multiple cancer types. There are 39 PPIs for PIK3CA, among which, 31 PPIs are potential oncoPPIs in at least one cancer type. We then click the "NETWORK VIEWER" button (Fig. 5a) to visualize these PPIs. PIK3CA-PIK3R3 is an oncoPPI in the highest number of cancer types (22 types). However, it lacked a PDB structure containing both interacting proteins to show the PPI interface mutations. PIK3CA-PIK3R1 are another potential oncoPPIs in 21 cancer types (second highest) having 86 interface mutations.

After clicking on the "P27986-P42336" button, MPM will load the information for PIK3CA-PIK3R1. Although both proteins were mutated in various cancer types, most of the interface mutations were detected on PIK3CA (Fig. 5b, orange bars). By exploring the "More" section, we also found that interface mutations of PIK3CA-PIK3R1 were associated with patient survival of COAD (Fig. $5 \mathrm{~d}, p=0.043$ ) and lung adenocarcinoma (LUAD) (Fig. 5e, $p=0.03$ ). Several drug responses are affected by the interface mutations. Dabrafenib is a competitive kinase inhibitor of BRAF for the treatment of melanoma [51]. It has been used in combination with trametinib for the treatment of nonsmall-cell lung cancer [52] and anaplastic thyroid cancer [53]. Dabrafenib had a higher $\mathrm{IC}_{50}$ in mutant tumor cell lines (Fig. $5 \mathrm{f}, p=0.0081$ ) compared to wild-type cell lines, consistent with a recent study that mutant PIK3CA and AKT3 increases the resistance of melanoma cells to BRAF inhibitor dabrafenib [54]. Epothilone B is a microtubule inhibitor used for the treatment of multiple myeloma [55]. It shows a lowered $\mathrm{IC}_{50}$ in mutant tumor cell lines (Fig. 5g, $p=0.04$ ). A previous study reported that epothilone B enhanced the apoptotic effects of ABT-737 through the PI3K/AKT/mTOR pathway [56]. Altogether, these findings could be used to guide the treatment for patients with interface mutations on PIK3CA-PIK3R1.

Next, we examined PIK3CA-PIK3R1 interface mutations that may be responsible for the COAD patients' survival using "Edgetic Mutation Explorer" (Fig. 5c). PDB accession 5XGJ was automatically selected, which contained both PIK3CA (chain A) and PIK3R1 (chain B). 5XGJ covered all the interface mutations, as indicated by the purple bars. For pan-cancer, several mutation hot-spots were revealed on PIK3CA: Glu545 (316 cases), Glu542 (183 cases), Gln546 (54 cases), Asn345 (48 cases), Cys420 (26 cases), and Glu453 (25 cases). Gly376 (13 cases) and Asn564 (10 cases) were the most frequently mutated spots. Some of these were reported previously in several cancer types [57-62]. We then set the cancer type to COAD. Glu545 (38 cases) and Glu542 (14 cases) of PIK3CA were the most populated mutation sites in COAD patients. Cys420 was highly mutated in pancancer (26 cases), and had 4 cases in COAD which were all mutated to arginine. By clicking Cys420, we also found that Cys420 on PIK3CA was directly pointing towards PIK3R1, with a distance less than 3.5 angstrom $(\AA)$ to the nearest residues on PIK3R1.

\section{Limitation and future directions}

We acknowledge several limitations. We assembled PPI interface data from known protein complex structures, homology models, and machine learning-based 
computational computation as the crystal structure-derived data is very limited. Although we showed that somatic missense mutations were significantly enriched in computationally predicted PPI interfaces [20], further improving the quality of PPI interfaces (including cryogenic electron microscopy (cryo-EM) structure) are highly needed in the future. The computation for SMEs did not take the sequence composition and amino acid specific mutation rate into consideration. However, when we recomputed the significance of the SMEs, we found that the new results are highly consistent to the original results, suggesting a small effect by accounting for these factors (Additional file 1: Fig. S4). Third, we applied a 15-amino acid window $( \pm 7)$ to screen for mutations for phosphorylation sites. Mutations that do not directly overlap with the functional sites may not have nodetic or edgetic effect. However, if we only consider the exact position (i.e., \pm 0 ), the analysis will be underpower due to the sparsity of data. In addition, we evaluated different window sizes $( \pm 0, \pm 1, \pm 3$, and \pm 5 ) for phosphorylation sites and found that $\pm 3, \pm 5$, and \pm 7 produced similar results (Additional file 1: Fig. S5). It is unclear whether the presence of the mutation may have a functional effect on phosphorylationbased singling networks. We tested how phosphorylation site mutations have functional impact on signal networks using proteogenomics data from The National Cancer Institute's Clinical Proteomic Tumor Analysis Consortium (CPTAC) using $\mathrm{COAD}$ as an example. We found that protein quantification is significantly lowered in mutated phosphorylation sites than wild type sites in $\operatorname{COAD}(p=0.012$, Additional file 1: Fig. S6); yet, protein quantification is relatively lowered in mutated phosphorylation sites than wild type sites in breast (BRCA, $p=0.052$ ) and ovarian cancer (OV, $p=0.18)$. The differences of protein quantification in wild type and mutated phosphorylation sites in BRCA and OV are not significant, which could be due to an insufficient number of tumor samples and overall low mutation load in BRCA and OV. These observations reveal potential functional impacts of phosphorylation site mutations; further experimental validation is highly warranted using large-scale proteogenomics and phosphoproteomics datasets from cancer cell lines or tumor tissues. Finally, the human interactome is still incomplete and PPIs may have literature bias. We will continue updating the human interactome into the database, especially including more unbiased systematic PPIs data [20]. In addition, we will offer functions for selecting smaller window sizes for the functional sites. Future updates for MPM will be focused on providing more complete, high-quality human interactome (including protein-DNA/RNA interactions as well), functional sites, and proteogenomics data from CPTAC. We will integrate more human genome sequencing data, including Trans-Omics for Precision Medicine (TOPMed) Program [63], Alzheimer's Disease Sequencing Project (ADSP) [64], and International Cancer Genome Consortium (ICGC) [65], to improve utilities of MPM by adding more personalized genome analyses.

\section{Conclusions}

In summary, My Personal Mutanome offers a comprehensive database and powerful visualization tools that bridge the translational gap between large-scale genomic medicine studies and clinical outcomes. MPM offers rapid searching of actionable mutations and targets to guide personalized treatments and precision medicine drug discovery. By 
mapping mutations to PPI interfaces and protein functional sites and integrating clinical responses in terms of patient survival and drug response, MPM helps users identify cancer-driving and actionable missense somatic mutations associated with nodetic or edgetic effects in the scope of human protein-protein interactome and provides mechanistic and potential drug treatment implications. MPM will be updated annually to continue to provide the most complete data available.

\section{Supplementary Information}

The online version contains supplementary material available at https://doi.org/10.1186/s13059-021-02269-3.

Additional file 1: Supplementary figure S1-S6. Figure S1. Diagram illustrating different types of nodetic versus edgetic perturbation leading to distinct phenotypes. Figure S2. Mutation rate is enriched in the ligand binding sites for these cancer types. Figure S3. Mutation rate is enriched in the phosphorylation sites for these cancer types. Figure $\mathbf{S 4}$. Correlation between the original results and new results by considering the amino acid specific background mutation rate and the amino acid composition for ligand-binding (LB) sites and posttranslational modification (PTM) sites across 33 cancer types. Figure S5. Comparison of the results using different window sizes for the phosphorylation sites across all cancer types. Figure S6. Protein quantification is lowered in mutated phosphorylation sites than wild type sites in (A) breast invasive carcinoma, (B) colon adenocarcinoma, and (C) ovarian serous cystadenocarcinoma.

Additional file 2. Review history.

\section{Acknowledgements}

The results published here are in whole or part based upon data generated by the TCGA Research Network: https:/portal.gdc. cancer.gov. We thank the Lerner Research Institute Computing Services for hosting My Personal Mutanome. C.E. is the Sondra J. and Steven R. Hardis Chair of Cancer Genomic Medicine at the Cleveland Clinic, and an ACS Clinical Research Professor.

\section{Review history}

The review history is available as Additional file 2.

\section{Peer review information}

Yixin Yao was the primary editor of this article and managed its editorial process and peer review in collaboration with the rest of the editorial team.

\section{Authors' contributions}

F.C. conceived the study. Y.Z. constructed the database and developed the website. J.Z., J.F., and Y.Z. performed the data gathering and processing. J.Z., Y.Z., and W.M. performed the data analyses. R.N., L.L., T.A.C., and C.E. discussed and interpreted all results. Y.Z., J.Z., and F.C. wrote the manuscript. All authors critically revised the manuscript and gave final approval.

\section{Funding}

This work was supported, in part, by the VeloSano Pilot Program (Cleveland Clinic Taussig Cancer Institute), by the National Heart, Lung, and Blood Institute of the National Institutes of Health (NIH) under Award Number R00 HL138272 and the National Institute of Aging under Award Number R01AG066707 and 3R01AG066707-01S1 to F.C. This work has been also supported in part with Federal funds from the Frederick National Laboratory for Cancer Research, National Institutes of Health, under contract HHSN261200800001E. This research was supported (in part) by the Intramural Research Program of NIH, Frederick National Lab, Center for Cancer Research. The content of this publication does not necessarily reflect the views or policies of the Department of Health and Human Services, nor does mention of trade names, commercial products, or organizations imply endorsement by the US Government.

\section{Availability of data and materials}

My Personal Mutanome is available at https://mutanomelerner.ccf.org to all users without any login or registration restrictions. The code for all mutation mapping and analysis can be found in https//github.com/ChengF-Lab/mutanome under the MIT License [66] and on Zenodo [67]. Gene and protein information was retrieved from HGNC (https:/uww.genenames.org/) [21] and UniProt (https:/www.uniprot.org/uniprot) [22]. PPI interface information was combined from three sources: PDB (http://www.rcsb.org/) [16], ECLAIR (http://interactomeinsider.yulab.org/) [17], and Interactome3D (https://interactome3dirbbarcelona.org/) [18]. Protein functional sites were downloaded from dbPTM (http://dbptm.mbc.nctu.edu.tw/) [24], PhosphoSitePlus (https://www.phosphosite. org/homeAction.action) [25], Phospho.ELM (http//phospho.elm.eu.org/) [26], PTMD (http//ptmd.biocuckoo.org/) [27], and BioLiP (https://zhanglab.ccmb.med.umich.edu/BioLiP/) [28]. Somatic mutation and cancer patient information was downloaded from TCGA GDC Data Portal (https://portal.gdc.cancer.gov/). Mutation scores were retrieved from https://cadd.gs.washington.edu/ [30] and http://www.muffunc.com/ [31]. Drug response data were retrieved from GDSC (http://www.cancerrxgene.org/) [32].

\section{Ethics approval and consent to participate}

Not applicable

\section{Consent for publication}

Not applicable 


\section{Competing interests}

The authors declare that they have no competing interests.

\section{Author details}

${ }^{1}$ Genomic Medicine Institute, Lerner Research Institute, Cleveland Clinic, Cleveland, OH 44195, USA. ${ }^{2}$ Department of Systems Biology, Herbert Irving Comprehensive Center, Columbia University, New York, NY 10032, USA. ${ }^{3}$ Department of Biomedical Informatics, Columbia University, New York, NY 10032, USA. ${ }^{4}$ Department of Biomedical Informatics, College of Medicine, Ohio State University, Columbus, OH 43210, USA. ${ }^{5}$ Computational Structural Biology Section, Basic Science Program, Frederick National Laboratory for Cancer Research, National Cancer Institute at Frederick, Frederick, MD 21702, USA. 6 Department of Human Molecular Genetics and Biochemistry, Sackler School of Medicine, Tel Aviv University, 69978 Tel Aviv, Israel. ' Center for Immunotherapy and Precision Immuno-Oncology, Lerner Research Institute, Cleveland Clinic, Cleveland, OH 44195, USA. ${ }^{8}$ Department of Molecular Medicine, Cleveland Clinic Lerner College of Medicine, Case Western Reserve University, Cleveland, OH 44195, USA. ${ }^{9}$ Case Comprehensive Cancer Center, Case Western Reserve University School of Medicine, Cleveland, $\mathrm{OH} 44106$, USA. ${ }^{10}$ Taussig Cancer Institute, Cleveland Clinic, Cleveland, OH 44195, USA. " Department of Genetics and Genome Sciences, Case Western Reserve University School of Medicine, Cleveland, OH 44106, USA.

Received: 31 May 2020 Accepted: 13 January 2021

Published online: 29 January 2021

\section{References}

1. Hudson TJ, Anderson W, Aretz A, Barker AD, Bell C, Bernabé RR, et al. International network of cancer genome projects. Nature. 2010;464:993-8

2. The Cancer Genome Atlas Research Network, Chang K, Creighton CJ, Davis C, Donehower L, Drummond J, et al. The Cancer Genome Atlas Pan-Cancer analysis project. Nat Genet. 2013;45:1113-20.

3. Tate JG, Bamford S, Jubb HC, Sondka Z, Beare DM, Bindal N, et al. COSMIC: the catalogue of somatic mutations in cancer. Nucleic Acids Res. 2019;47:D941-7.

4. Cerami E, Gao J, Dogrusoz U, Gross BE, Sumer SO, Aksoy BA, et al. The cBio Cancer Genomics Portal: an open platform for exploring multidimensional cancer genomics data. Cancer Discov. 2012;2:401-4.

5. Gao J, Aksoy BA, Dogrusoz U, Dresdner G, Gross B, Sumer SO, et al. Integrative analysis of complex cancer genomics and clinical profiles using the cBioPortal. Sci Signal. 2013;6:pl1.

6. Nussinov R, Jang H, Tsai CJ, Cheng F. Review: precision medicine and driver mutations: computational methods, functional assays and conformational principles for interpreting cancer drivers. PLoS Comput Biol. 2019;15:e1006658.

7. Cheng F, Liang H, Butte AJ, Eng C, Nussinov R. Personal mutanomes meet modern oncology drug discovery and precision health. Pharmacol Rev. 2019;71:1-19.

8. Vidal M, Cusick ME, Barabási AL. Interactome networks and human disease. Cell. 2011;144:986-98.

9. Sahni N, Yi S, Zhong Q, Jailkhani N, Charloteaux B, Cusick ME, et al. Edgotype : the link between genotype and phenotype. Curr Opin Genet Dev. 2013;23:649-57.

10. Sahni N, Yi S, Taipale M, Fuxman Bass Jl, Coulombe-Huntington J, Yang F, et al. Widespread macromolecular interaction perturbations in human genetic disorders. Cell. 2015;161:647-60.

11. Cheng F, Jia P, Wang Q, Lin CC, Li WH, Zhao Z. Studying tumorigenesis through network evolution and somatic mutational perturbations in the cancer interactome. Mol Biol Evol. 2014;31:2156-69.

12. Zhao J, Cheng F, Wang Y, Arteaga CL, Zhao Z. Systematic prioritization of druggable mutations in $\sim 5000$ genomes across 16 cancer types using a structural genomics-based approach. Mol Cell Proteomics. 2016;15:642-56.

13. Shen Q, Cheng F, Song H, Lu W, Zhao J, An X, et al. Proteome-scale investigation of protein allosteric regulation perturbed by somatic mutations in 7,000 cancer genomes. Am J Hum Genet. 2017;100:5-20.

14. Zhao J, Cheng F, Zhao Z. Tissue-specific signaling networks rewired by major somatic mutations in human cancer revealed by proteome-wide discovery. Cancer Res. 2017;77:2810-21.

15. Wang X, Wei $X$, Thijssen B, Das J, Lipkin SM, Yu H. Three-dimensional reconstruction of protein networks provides insight into human genetic disease. Nat Biotechnol. 2012;30:159-64.

16. Berman HM, Westbrook J, Feng Z, Gilliland G, Bhat TN, Weissig H, et al. The protein data bank. Nucleic Acids Res. 2000;28:235-42.

17. Meyer MJ, Beltrán JF, Liang S, Fragoza R, Rumack A, Liang J, et al. Interactome INSIDER: a structural interactome browser for genomic studies. Nat Methods. 2018;15:107-14.

18. Mosca R, Céol A, Aloy P. Interactome3D: adding structural details to protein networks. Nat Methods. 2013;10:47-53.

19. Bouhaddou M, Eckhardt M, Chi Naing ZZ, Kim M, Ideker T, Krogan NJ. Mapping the protein-protein and genetic interactions of cancer to guide precision medicine. Curr Opin Genet Dev. 2019;54:110-7.

20. Cheng F, Zhao J, Wang Y, Lu W, Liu Z, Zhou Y, et al. Comprehensive characterization of protein-protein interactions perturbed by disease mutations. Nat Genet. in press, 2021. https://doi.org/10.1038/s41588-020-00774-y.

21. Yates B, Braschi B, Gray KA, Seal RL, Tweedie S, Bruford EA. Genenames.org: the HGNC and VGNC resources in 2017. Nucleic Acids Res. 2017;45:D619-25.

22. UniProt Consortium. UniProt: a worldwide hub of protein knowledge. Nucleic Acids Res. 2018;47:D506-15.

23. Cheng F, Kovács IA, Barabási AL. Network-based prediction of drug combinations. Nat Commun. 2019;10:1197.

24. Huang KY, Lee TY, Kao HJ, Ma CT, Lee CC, Lin TH, et al. dbPTM in 2019: exploring disease association and cross-talk of post-translational modifications. Nucleic Acids Res. 2019;47:D298-308.

25. Hornbeck PV, Zhang B, Murray B, Kornhauser JM, Latham V, Skrzypek E. PhosphoSitePlus, 2014: mutations, PTMs and recalibrations. Nucleic Acids Res. 2015;43:D512-20.

26. Dinkel H, Chica C, Via A, Gould CM, Jensen L, Gibson TJ, et al. Phospho.ELM: a database of phosphorylation sitesupdate 2011. Nucleic Acids Res. 2011;39:D261-7.

27. Xu H, Wang Y, Lin S, Deng W, Peng D, Cui Q, et al. PTMD: a database of human disease-associated post-translational modifications. Genomics Proteomics Bioinformatics. 2018;16:244-51. 
28. Yang J, Roy A, Zhang Y. BioLiP: a semi-manually curated database for biologically relevant ligand-protein interactions. Nucleic Acids Res. 2013;41:D1096-103.

29. Wang K, Li M, Hakonarson H. ANNOVAR: functional annotation of genetic variants from high-throughput sequencing data. Nucleic Acids Res. 2010;38:e164.

30. Rentzsch P, Witten D, Cooper GM, Shendure J, Kircher M. CADD: predicting the deleteriousness of variants throughout the human genome. Nucleic Acids Res. 2019:47:D886-94

31. Wagih $O$, Galardini M, Busby BP, Memon D, Typas A, Beltrao P. A resource of variant effect predictions of single nucleotide variants in model organisms. Mol Syst Biol. 2018;14:e8430.

32. Yang W, Soares J, Greninger P, Edelman EJ, Lightfoot H, Forbes S, et al. Genomics of Drug Sensitivity in Cancer (GDSC): a resource for therapeutic biomarker discovery in cancer cells. Nucleic Acids Res. 2013;41:D955-61.

33. Franz M, Lopes CT, Huck G, Dong Y, Sumer O, Bader GD. Cytoscape.js: a graph theory library for visualisation and analysis. Bioinformatics. 2016;32:309-11.

34. Biasini M. pv: V1.8.1.. Zenodo. 2015. Available from: https://github.com/biasmv/pv/tree/v1.8.1. Accessed 9 Apr 2019.

35. Colicelli J. Human RAS superfamily proteins and related GTPases. Sci STKE. 2004;2004:RE13.

36. Neumann J, Zeindl-Eberhart E, Kirchner T, Jung A. Frequency and type of KRAS mutations in routine diagnostic analysis of metastatic colorectal cancer. Pathol Res Pract. 2009;205:858-62.

37. Biankin AV, Waddell N, Kassahn KS, Gingras MC, Muthuswamy LB, Johns AL, et al. Pancreatic cancer genomes reveal aberrations in axon guidance pathway genes. Nature. 2012;491:399-405.

38. Collisson EA, Campbell JD, Brooks AN, Berger AH, Lee W, Chmielecki J, et al. Comprehensive molecular profiling of lung adenocarcinoma: the cancer genome atlas research network. Nature. 2014;511:543-50.

39. Ihle NT, Byers LA, Kim ES, Saintigny P, Lee JJ, Blumenschein GR, et al. Effect of KRAS oncogene substitutions on protein behavior: implications for signaling and clinical outcome. J Natl Cancer Inst. 2012;104:228-39.

40. Chen CC, Er TK, Liu YY, Hwang JK, Barrio MJ, Rodrigo M, et al. Computational analysis of KRAS mutations: implications for different effects on the KRAS p.G12D and p.G13D mutations. Plos One. 2013;8:e55793.

41. Margonis GA, Kim Y, Spolverato G, Ejaz A, Gupta R, Cosgrove D, et al. Association between specific mutations in KRAS codon 12 and colorectal liver metastasis. JAMA Surg. 2015;150:722-9.

42. Di Magliano MP, Logsdon CD. Roles for KRAS in pancreatic tumor development and progression. Gastroenterology. 2013;144:1220-9.

43. Waters AM, Der CJ. KRAS: the critical driver and therapeutic target for pancreatic cancer. Cold Spring Harb Perspect Med. 2018;8:a031435.

44. Salama AKS, Kim KB. Trametinib (GSK1 120212) in the treatment of melanoma. Expert Opin Pharmacother. 2013;14:619-27.

45. Blumenschein GR, Smit EF, Planchard D, Kim DW, Cadranel J, De Pas T, et al. A randomized phase II study of the MEK1/ MEK2 inhibitor trametinib (GSK1120212) compared with docetaxel in KRAS-mutant advanced non-small-cell lung cancer (NSCLC). Ann Oncol. 2015;26:894-901.

46. Gadgeel SM, Miao J, Riess JW, Mack PC, Gerstner GJ, Burns TF, et al. S1507: phase II study of docetaxel and trametinib in patients with G12C or non-G12C KRAS mutation positive (+) recurrent non-small cell lung cancer (NSCLC). J Clin Oncol. 2019:37:9021.

47. Pao W, Miller V, Zakowski M, Doherty J, Politi K, Sarkaria I, et al. EGF receptor gene mutations are common in lung cancers from "never smokers" and are associated with sensitivity of tumors to gefitinib and erlotinib. Proc Natl Acad Sci U S A. 2004;101:13306-11.

48. Pao W, Wang TY, Riely GJ, Miller VA, Pan Q, Ladanyi M, et al. KRAS mutations and primary resistance of lung adenocarcinomas to gefitinib or erlotinib. PLoS Med. 2005;2:0057-61.

49. Cantley LC. The phosphoinositide 3-kinase pathway. Science. 2002;296:1655-7.

50. Vallejo-Díaz J, Chagoyen M, Olazabal-Morán M, González-García A, Carrera AC. The opposing roles of PIK3R1/p85a and PIK3R2/p85 $\beta$ in cancer. Trends Cancer. 2019;5:233-44.

51. Gibney GT, Zager JS. Clinical development of dabrafenib in BRAF mutant melanoma and other malignancies. Expert Opin Drug Metab Toxicol. 2013;9:893-9.

52. Planchard D, Besse B, Groen HJM, Souquet PJ, Quoix E, Baik CS, et al. Dabrafenib plus trametinib in patients with previously treated BRAFV600E-mutant metastatic non-small cell lung cancer: an open-label, multicentre phase 2 trial. Lancet Oncol. 2016:17:984-93.

53. Subbiah V, Cabanillas ME, Kreitman RJ, Wainberg ZA, Cho JY, Keam B, et al. Dabrafenib and trametinib treatment in patients with locally advanced or metastatic BRAF V600-mutant anaplastic thyroid cancer. J Clin Oncol. 2018;36:7-13.

54. Irvine M, Stewart A, Pedersen B, Boyd S, Kefford R, Rizos H. Oncogenic PI3K/AKT promotes the step-wise evolution of combination BRAF/MEK inhibitor resistance in melanoma. Oncogenesis. 2018;7:72.

55. Lin B, Catley L, LeBlanc R, Mitsiades C, Burger R, Tai YT, et al. Patupilone (epothilone B) inhibits growth and survival of multiple myeloma cells in vitro and in vivo. Blood. 2005;105:350-7.

56. Li Y, Sun J, Hu X, Pan Y, Yan W, Li Q, et al. Epothilone B induces apoptosis and enhances apoptotic effects of ABT-737 on human cancer cells via PI3K/AKT/mTOR pathway. J Cancer Res Clin Oncol. 2016;142:2281-9.

57. Gymnopoulos M, Elsliger MA, Vogt PK. Rare cancer-specific mutations in PIK3CA show gain of function. Proc Natl Acad Sci U S A. 2007;104:5569-74.

58. Murugan AK, Thi Hong N, Fukui Y, Munirajan AK, Tsuchida N. Oncogenic mutations of the PIK3CA gene in head and neck squamous cell carcinomas. Int J Oncol. 2008;32:101-11.

59. Janku F, Wheler JJ, Naing A, Stepanek VM, Falchook GS, Fu S, et al. PIK3CA mutations in advanced cancers: characteristics and outcomes. Oncotarget. 2012;3:1566-75.

60. Chen L, Yang L, Yao L, Kuang XY, Zuo WJ, Li S, et al. Characterization of PIK3CA and PIK3R1 somatic mutations in Chinese breast cancer patients. Nat Commun. 2018;9:1357.

61. Jiang W, He T, Liu S, Zheng Y, Xiang L, Pei X, et al. The PIK3CA E542K and E545K mutations promote glycolysis and proliferation via induction of the $\beta$-catenin/SIRT3 signaling pathway in cervical cancer. J Hematol Oncol. 2018;11:139.

62. Bailey MH, Tokheim C, Porta-Pardo E, Sengupta S, Bertrand D, Weerasinghe A, et al. Comprehensive characterization of cancer driver genes and mutations. Cell. 2018:173:371-385.e18

63. Sarnowski C, Leong A, Raffield LM, Wu P, de Vries PS, DiCorpo D, et al. Impact of rare and common genetic variants on diabetes diagnosis by hemoglobin A1c in multi-ancestry cohorts: the trans-omics for precision medicine program. Am J Hum Genet. 2019;105:706-18. 
64. Beecham GW, Bis JC, Martin ER, Choi S-H, DeStefano AL, van Duijn CM, et al. The Alzheimer's disease sequencing project: study design and sample selection. Neurol Genet. 2017;3:e194.

65. ICGC/TCGA Pan-Cancer Analysis of Whole Genomes Consortium. Pan-cancer analysis of whole genomes. Nature. 2020;578:82-93.

66. Zhou Y, Zhao J, Fang J, Martin W, Li L, Nussinov R, et al. ChengF-Lab/mutanome v1.1. Github 2021. Available from https://github.com/ChengF-Lab/mutanome. Accessed 12 Oct 2020.

67. Zhou Y, Zhao J, Fang J, Martin W, Li L, Nussinov R, et al. ChengF-Lab/mutanome v1.1. Zenodo; 2021. https://doi.org/10. 5281/zenodo.4416655.

\section{Publisher's Note}

Springer Nature remains neutral with regard to jurisdictional claims in published maps and institutional affiliations.

Ready to submit your research? Choose BMC and benefit from:

- fast, convenient online submission

- thorough peer review by experienced researchers in your field

- rapid publication on acceptance

- support for research data, including large and complex data types

- gold Open Access which fosters wider collaboration and increased citations

- maximum visibility for your research: over $100 \mathrm{M}$ website views per year

At $\mathrm{BMC}$, research is always in progress.

Learn more biomedcentral.com/submissions 